\title{
NA48 results on semileptonic decays and extraction of |Vus|
}

\section{Andreas Winhart ${ }^{* \dagger}$}

Universitaet Mainz, Germany

E-mail: andreas.winhartecern.ch

The NA48 experiment at the CERN SPS was originally designed and used for the precision measurement of direct CP violation in kaon decays. Moreover, a large number of measurements of rare kaon and hyperon decays have been carried out. All four analyses presented here are based on the same data sample, taken in September 1999 during a special run with pure $K_{L}$ beam. A simple minimum bias trigger was set up to accept only events with two charged tracks, adequate to collect semileptonic $K_{L}$ decays. During two days of data taking about 80 million 2-track events were recorded.

We measured the branching ratio $R$ of the decay $K_{L} \rightarrow \pi^{ \pm} e^{\mp} v$ relative to all charged $K_{L}$ decays with two tracks: $R=0.4978 \pm 0.0035$. From this we derived the $K_{e 3}$ branching fraction $B R\left(K_{e 3}\right)=0.4010 \pm 0.0028_{\text {exp. }} \pm 0.0035_{\text {norm. }}$ and extracted the CKM parameter $|V u s|=$ $0.2187 \pm 0.0016_{\text {exp. }} \pm 0.0023_{\text {theo. }}$. The uncertainty on $|V u s|$ is dominated by the insufficient knowledge of the vector form factor $f_{+}^{K^{0}} \pi^{-}$.

From a sample of 18977 reconstructed $K_{e 3 \gamma}^{0}$ and 5.6 million $K_{e 3}^{0}$ events we measured the relative branching ratio $B R\left(K_{L} \rightarrow \pi^{ \pm} e^{\mp} v \gamma / K_{L} \rightarrow \pi^{ \pm} e^{\mp} v\right)=\left(0.964 \pm 0.008_{-0.009}^{+0.011}\right) \%$.

The same $K_{e 3}^{0}$ sample was used to measure the Dalitz plot density. A linear $q^{2}$ dependence of the vector form factor was found. There is no evidence for scalar or tensor couplings. Assuming pure vector coupling, the result for the slope parameter is $\lambda_{+}=0.0288 \pm 0.0004_{\text {stat. }} \pm 0.0011_{\text {syst }}$.

In a similar way the Dalitz plot density for $K_{L} \rightarrow \pi^{ \pm} \mu^{\mp} v_{e}$ events is being studied. Based on 2.64 million reconstructed $\mathrm{K}_{\mu 3}^{0}$ decays in data, we obtain the preliminary results $\lambda_{+}=$ $0.0260 \pm 0.0007_{\text {stat. }} \pm 0.0010_{\text {syst. }}$ and $\lambda_{0}=0.0120 \pm 0.0008_{\text {stat. }} \pm 0.0015_{\text {syst. }}$.

International Europhysics Conference on High Energy Physics

July 21st - 27th 2005

Lisboa, Portugal

\footnotetext{
* Speaker.

†n behalf of the NA48 collaboration: Cagliari, Cambridge, CERN, Dubna, Edinburgh, Ferrara, Firenze, Mainz, Orsay, Perugia, Pisa, Saclay, Siegen, Torino, Warsaw, Wien.
} 


\section{The NA48 detector}

One of the main components of the NA48 detector is the electromagnetic calorimeter based on liquid krypton with tower readout. The calorimeter is 27 decay lengths long and fully contains electromagnetic showers with energies up to $100 \mathrm{GeV}$. The energy resolution obtained is $\sigma(E) / E=$ $(3.2 / \sqrt{E} \oplus 9.0 / E \oplus 0.42) \%$, with $E$ in $\mathrm{GeV}$. A magnetic spectrometer, consisting of four drift chambers and a central dipole magnet is used to measure the momenta of charged particles. The spatial resolution per projection is $100 \mu \mathrm{m}$ and the momentum resolution is given by $\sigma(p) / p=$ $(0.48 \oplus 0.009 p) \%$, with $p$ in $\mathrm{GeV} / \mathrm{c}$. A detailed description of the apparatus can be found in [2].

\section{2. $\mathbf{K}_{\mathbf{L}} \rightarrow \pi^{ \pm} \mathbf{e}^{\mp} v_{\mathbf{e}}\left(\mathbf{K}_{\mathbf{e} 3}^{\mathbf{0}}\right)$ branching ratio and extraction of $\left|\mathbf{V}_{\mathbf{u s}}\right|$}

The unitary condition for the first row of the CKM quark mixing matrix is at present fulfilled only at the $10 \%$ C. L. [1]. This has renewed interest in the measurement of the coupling constant $V_{u s}$ for strangeness-changing weak transitions. The most precise information on $V_{u s}$ comes from the decay $K_{L} \rightarrow \pi^{ \pm} e^{\mp} v$, which is a pure vector transition.

The basic idea in this experiment is to measure the $K_{e 3}$ signal relative to as many other channels as possible. The data sample from the minimum bias run is well suited, as one can normalize to all decays with two charged particles in the final state (2T), mainly $\pi e v, \pi \mu v, \pi^{+} \pi^{-} \pi^{0}, \pi^{+} \pi^{-}$and $3 \pi^{0}$ with Dalitz decay of one $\pi^{0}$. This ratio $R$ is given as:

$$
R=\frac{\Gamma\left(K_{L} \rightarrow \pi e v\right)}{\Gamma\left(K_{L} \rightarrow \text { all } 2 \text {-track events }\right)}=\frac{N_{k e 3} / a_{k e 3}}{N_{2 T} / a_{2 T}},
$$

with $N_{i}$ and $a_{i}$ being the number of reconstructed events and the acceptances, respectively. Since the neutral decay modes have been measured and are dominated by the decay $K_{L} \rightarrow \pi^{0} \pi^{0} \pi^{0}\left(3 \pi^{0}\right)$, the sum of branching ratios of all $K_{L}$ decay modes with two charged tracks $B R(2 T)$ is known [1]:

$$
B R(2 T)=1-B R\left(3 \pi^{0}\right) \pm \text { small corrections }=1.0048-B R\left(3 \pi^{0}\right) \quad(\approx 80 \%) .
$$

The $K_{e 3} \mathrm{BR}$ can be obtained from the ratio $R: B R\left(K_{e 3}\right)=R \times B R(2 T)$.

A set of cuts was applied to select good 2-track events ( $\sim 12.6$ million). The $K_{e 3}$ signal was extracted from this sample by a single additional criterion, that at least one track should be consistent with an electron. This was done by requiring that the ratio $E / p$ exceed 0.93 , where $E$ is the measured energy in the calorimeter and $p$ is the measured momentum in the magnetic spectrometer. 6.759 million $K_{e 3}$ events were accepted.

The detector response was reproduced using a GEANT based Monte Carlo simulation. The average 2-track acceptance was obtained from a weighted mean of the individual acceptances. The results for $B R(2 T)=(80.56 \pm 0.70) \%$ (the uncertainty coming from $B R\left(K_{L} \rightarrow 3 \pi^{0}\right)=(19.92 \pm$ $0.70) \%$, averaged between PDG [1] and the latest $\mathrm{KTeV}$ result [3]) and $R=0.4978 \pm 0.0035$ lead to the follwing $K_{e 3}$ branching ratio:

$$
B R\left(K_{e 3}\right)=0.4010 \pm 0.0028 \pm 0.0035,
$$

with the first error being the complete experimental error and the second the external error from the normalization. This value exceeds the 2004 world average [1] by 3.4\%, but is in agreement with recent measurements from KTeV [2] and KLOE [5]. 
The CKM matrix element $\left|V_{u s}\right|$ can be extracted from the $K_{e 3}^{0}$ decay parameters by ref. [4]

$$
\left|V_{u s}\right|=\sqrt{\frac{128 \pi^{3} \Gamma\left(K_{e 3}^{0}\right)}{G_{F}^{2} M_{K^{0}}^{5} S_{E W} I_{K^{0}}}} \frac{1}{f_{+}^{K^{0} \pi^{-}}} .
$$

Three quantities in this equation are taken from theory. $S_{E W}$ is the short-distance radiative correction factor, $I_{K^{0}}$ is the phase space integral and $f_{+}^{K^{0} \pi^{-}}$is the form factor at zero momentum transfer. To determine $\left|V_{u s}\right|$ we followed the prescription and used the numerical results in ref. [4], obtaining $\left|V_{u s}\right| f_{+}(0)=0.2146 \pm 0.0016$. Taking the value $f_{+}(0)=0.981 \pm 0.010$ [4] we get

$$
\left|V_{u s}\right|=0.2187 \pm 0.0016_{\text {exp. }} \pm 0.0023_{\text {theo. }} .
$$

This is still 2.4 sigma lower than required by the 3-generation unitarity of the CKM matrix, but the result is dominated by theoretical uncertainties and strongly depends on the choice of $f_{+}(0)$. A more detailed description of the analysis can be found in [6].

\section{Radiative $\mathbf{K}_{\mathrm{e} 3}^{\mathbf{0}}$ branching ratio $\mathrm{K}_{\mathrm{L}} \rightarrow \pi^{ \pm} \mathbf{e}^{\mp} v_{\mathrm{e}} \gamma$}

The study of radiative semileptonic decays gives information about the structure of the decaying particle and allows to test models describing hadron interactions at small momentum transfer $(C h P T)$. We measured the relative branching ratio $K_{L} \rightarrow \pi^{ \pm} e^{\mp} v \gamma / K_{L} \rightarrow \pi^{ \pm} e^{\mp} v$. All events were selected by the same criteria, except for $K_{e 3 \gamma}^{0}$ events we required in addition one hard $\gamma$ in the electromagnetic calorimeter. For this measurement it is mandatory to take radiative corrections into account properly. For this purpose Monte Carlo events were generated with the PHOTOS package [7]. In a second step the events were weighted to fit the data in the variable $\Theta_{e \gamma}^{*}$, the angle between the electron and the photon in the kaon c.m., thus fine-tuning the description of radiative corrections effects. A very good agreement between data and $\mathrm{MC}$ has been found for all variables. With 18977 reconstructed $K_{e 3 \gamma}^{0}$ and $5.594 \times 10^{6} K_{e 3}^{0}$ events we obtain:

$$
R=\frac{\Gamma\left(K_{L} \rightarrow \pi e v \gamma, E_{\gamma}^{*}>30 \mathrm{MeV}, \Theta_{e \gamma}^{*}>20^{\circ}\right)}{\Gamma\left(K_{L} \rightarrow \pi e v\right)}=\left(0.964 \pm 0.008_{-0.009}^{+0.011}\right) \% .
$$

The result is in good agreement with theoretical predictions and recent ChPt calculations. A detailed description of the analysis is given in [8].

\section{4. $\mathbf{K}_{\mathrm{L}} \rightarrow \pi^{ \pm} \mathbf{e}^{\mp} v_{\mathbf{e}}$ form factors}

Assuming that only the vector coupling contributes to the decay, the matrix element can be written in terms of two dimensionless form factors $f_{ \pm}(t)$ :

$$
M=G_{F} / \sqrt{2} V_{u s}\left[f_{+}(t)\left(P_{K}+P_{\pi}\right)^{\mu} \bar{u}_{l} \gamma_{\mu}\left(1+\gamma_{5}\right) u_{v}+f_{-}(t) m_{l} \bar{u}_{l}\left(1+\gamma_{5}\right) u_{v}\right],
$$

where $P_{K}$ and $P_{\pi}$ are the kaon and pion four-momenta, $\bar{u}_{l}$ and $u_{v}$ are the lepton spinors and $m_{l}$ is the lepton mass. The contribution of $f_{-}$is proportional to the lepton mass squared, and thus can be neglected in $K_{e 3}^{0}$ decays. The determination of the form factors is based on the measurement of the 
Dalitz plot density:

$$
\rho\left(E_{e}^{*}, E_{\pi}^{*}\right)=\frac{d N^{2}\left(E_{e}^{*}, E_{\pi}^{*}\right)}{d E_{e}^{*} d E_{\pi}^{*}} \propto f_{+}^{2}(t) \cdot A,
$$

with $A$ being a kinematical term and $E_{e}^{*}$ and $E_{\pi}^{*}$ are the electron and pion energies in the kaon c.m., respectively. It is usually assumed that the vector form factor depends linearly on $q^{2}$ (the square of the four-momentum transferred to the lepton system): $f_{+}\left(q^{2}\right)=f_{+}(0)\left(1+\lambda_{+} q^{2} / m_{\pi}^{2}\right)$.

Admitting all possible Lorentz-covariant couplings, we measured the form factors for vector $\left(f_{+}\left(q^{2}\right)\right)$, scalar $\left(f_{S}\right)$ and tensor $\left(f_{T}\right)$ interactions as well as the $q^{2}$ dependence of the vector form factor. The result is based on a sample of 5.6 million $K_{e 3}^{0}$ events:

$$
\begin{aligned}
\lambda_{+} & =0.0284 \pm 0.0007_{\text {stat. }} \pm 0.0013_{\text {syst. }} \\
\left|\frac{f_{S}}{f_{+}(0)}\right| & =0.015_{-0.010 \text { stat. }}^{+0.007} \pm 0.012_{\text {syst. }} \\
\left|\frac{f_{T}}{f_{+}(0)}\right| & =0.05_{-0.04 \text { stat. }}^{+0.03} \pm 0.03_{\text {syst. }} .
\end{aligned}
$$

There is no indication for scalar and tensor couplings. Assuming only V-A currents and a linear $q^{2}$ dependence we get

$$
\lambda_{+}=0.0288 \pm 0.0004_{\text {stat. }} \pm 0.0011_{\text {syst. }} .
$$

We find no evidence for a quadratic term $\lambda_{+}^{\prime}$. However, a fit to a dipole form factor yields a pole mass of $859 \pm 18 \mathrm{MeV}$, consistent with the $\mathrm{K}^{*}(892)$ mass. The analysis is described in detail in [9].

\section{5. $\mathbf{K}_{\mathbf{L}} \rightarrow \pi^{ \pm} \mu^{\mp} v_{\mathbf{e}}$ form factors}

Due to the much higher lepton mass the contribution from the form factor $f_{-}$becomes relevant. $f_{-}$is usually expressed in terms of the scalar form factor $f_{0}\left(q^{2}\right)=f_{+}\left(q^{2}\right)+q^{2} /\left(m_{K}^{2}+m_{\pi}^{2}\right) \cdot f_{-}\left(q^{2}\right)$ (with slope parameter $\lambda_{0}$ ). The form factors are determined by fitting the corrected data to a Dalitz plot density parametrisation, similar to the $K_{e 3}^{0}$ measurement. Radiative effects were properly simulated in the Monte Carlo, including real and virtual diagrams.

Based on 2.64 million reconstructed $\mathrm{K}_{\mu 3}^{0}$ decays in data, we obtain the preliminary results:

$$
\begin{aligned}
& \lambda_{+}=0.0260 \pm 0.0007_{\text {stat. }} \pm 0.0010_{\text {syst. }} . \\
& \lambda_{0}=0.0120 \pm 0.0008_{\text {stat. }} \pm 0.0015_{\text {syst. }} .
\end{aligned}
$$

\section{References}

[1] S. Eidelmann et al. (Particle Data Group), Phys. Lett. B 592, (2004) 1

[2] A. Lai et al., NA48 Collaboration, Eur. Physics J. C 22, (2001) 231-254

[3] T. Alexopoulos et al., KTeV collaboration, hep-ex/0406002

[4] V. Cirigliano, H. Neufeld and H. Pichl, Eur. Phys. J. C 35, (2004) 53

[5] F. Ambrosino et al., KLOE collaboration, hep-ex/0508027

[6] A. Lai et al., NA48 Collaboration, Phys. Lett. B 602, (2004) 41-51

[7] E. Barberio and Z. Was, Comput. Phys. Com. 79: 291-308 (1994)

[8] A. Lai et al., NA48 Collaboration, Phys. Lett. B 605, (2005) 247-255

[9] A. Lai et al., NA48 Collaboration, Phys. Lett. B 604, (2004) 1-10 